\title{
Comparison of eDNA metabarcoding to camera trapping for terrestrial vertebrate monitoring highlights the importance of substrate type, frequency of sampling and animal size
}

\author{
Ethan Ryan ${ }^{1}$, Phil Bateman ${ }^{1}$, Mieke van der Heyde ${ }^{1}$, Kristen Fernandes ${ }^{2}$, and Paul Nevill ${ }^{1}$ \\ ${ }^{1}$ Curtin University Bentley Campus \\ ${ }^{2}$ Curtin University
}

November 3, 2020

\begin{abstract}
Fauna biodiversity assessments often rely on traditional biomonitoring techniques such as camera traps, which may have biases that lead to gaps in biodiversity data. Environmental DNA (eDNA) has emerged as a new source of biodiversity data that may account for these gaps. However, eDNA biodiversity assessment remains relatively untested in terrestrial environments. We compared vertebrate detections using two independent monitoring methods: camera traps and eDNA ( $\mathrm{n}=160)$, across two sites in south-western Australia. We also investigated the suitability of tree hollow sediment as a source of eDNA, and the effect of other factors (visitation frequency and timing, animal size) on vertebrate species detectability. We detected 31 taxa with eDNA and 47 with camera traps of which 14 overlapped (12 mammals and 2 birds). Tree hollow sediment detected a wider range of biodiversity than did soil at the entrance of the hollow. By comparing camera trap data with eDNA sequence reads, we were able to detect animals with eDNA that had visited the area up to two months prior to sample collection, with a negative correlation between sequence read amount and days since last recorded detection via camera. "Large" animals ( $>3 \mathrm{~kg}$ ) detected via camera were associated with significantly higher sequence read amounts than smaller animals. Our results show the effect of substrate selection, frequency of sampling and animal size, on eDNA based surveys. If the aim is to detect broad taxon diversity eDNA based approaches need to be complemented by traditional vertebrate survey methods.
\end{abstract}

\section{Hosted file}

MER Manuscript 221020.pdf available at https://authorea.com/users/372664/articles/490605comparison-of-edna-metabarcoding-to-camera-trapping-for-terrestrial-vertebratemonitoring-highlights-the-importance-of-substrate-type-frequency-of-sampling-and-animalsize 